\title{
Die Gesetzentwürfe des Deutschen Richterbundes und der Neuen Richtervereinigung zur Selbstverwaltung der Justiz - Ein Vergleich im Überblick
}

Sowohl die NRV als auch der DRB haben aktuelle Gesetzentwürfe zur Selbstverwaltung der Justiz vorgelegt. Die Entwürfe kann man sich im Internet von der Homepage der NRV bzw. des DRB herunterladen. Der „Modellentwurf eines Landesjustizverwaltungsgesetzes“ des DRB stammt vom 25.3.2010. Der „Diskussionsentwurf“ der NRV besteht aus zwei Teilen (Gesetz zur Änderung des Grundgesetzes und Gesetz zur Änderung einfachen Rechts) und stammt vom 16.3.2010. Am „Diskussionsentwurf“ der NRV wird verbandsintern weiter gearbeitet; es ist allerdings davon auszugehen, dass sich an den für diese Analyse maßgeblichen Grundstrukturen des Entwurfs nichts wesentliches ändern wird.

Im folgenden sollen die Grundzüge der Entwürfe miteinander verglichen werden. Unter Ziffer 1 werden wesentliche Gemeinsamkeiten herausgestellt; Ziff. 2, 3, 4 und 5 enthalten die wichtigsten Unterschiede in den Vorstellungen der Verbände. Die vergleichende Darstellung soll sich auf die Grundstrukturen der beiden Entwürfe beschränken. Die (im NRV-Entwurf berücksichtigte) Selbstverwaltung der Staatsanwaltschaften wird im folgenden nicht berücksichtigt, da insoweit ein Gegenstück im Entwurf des DRB fehlt.

\section{Gemeinsamkeiten der Entwürfe von NRV und DRB}

Neue Richtervereinigung und Deutscher Richterbund stimmen in ihren Entwürfen grundsätzlich insoweit vollständig überein, als im Einklang mit der Verfassung der Bundesrepublik Deutschland, unter Berücksichtigung der maßgeblichen Empfehlungen des Europarats und im Hinblick auf die Entwicklung in nahezu allen europäischen Ländern seit 1948 der Einfluss der Exekutive auf die Ernennung, Beförderung, Versetzung und Disziplinierung von Richterinnen und Richtern so weit wie möglich ausgeschlossen werden soll. Ohne eine solche Veränderung kann nicht von einer tatsächlich unabhängigen Rechtsprechung im Sinne des Grundgesetzes gesprochen werden.

a) Die Unabhängigkeit der (einzelnen) Richter und die Unabhängigkeit der Justiz (als Organisation) sind die wichtigsten Voraussetzungen dafür, dass Richter sich bei ihren Entscheidungen nur am Gesetz orientieren. Die Unabhängigkeit soll sachfremde Einflüsse, insbesondere direkte oder indirekte Einflussnahmen der Politik, auf richterliche Entscheidungen ausschließen. Die Unabhängigkeit ist notwendige und wichtigste Bedingung für die Gewährleistung des Prinzips der Gesetzesbindung bei richterlichen Entscheidungen.

b) Das Verhalten und die Entscheidungen von Richtern werden nicht nur durch mögliche direkte Einwirkungen im Einzelfall (Anweisungen, Empfehlungen, Ratschläge oder Drohungen) beeinflusst, sondern vor allem durch organisatorische Maßnahmen. Ein Organisationssystem bewirkt immer in gewissem Umfang ein Anpassungsverhalten 

verwaltung der Justiz - Ein Vergleich im Überblick

der Richter, die der Organisation unterworfen sind. Das wird besonders deutlich bei der Auswahl und Ernennung von Richtern, bei Beförderungen und Beurteilungen, bei Versetzungen und bei der Disziplinierung von Richterinnen und Richtern.

c) Die generelle Bedeutung von Organisationsstrukturen für Richterliche Unabhängigkeit und Gesetzesbindung einerseits oder Anpassungsverhalten von Richtern andererseits (s. o. b)) ist sowohl nach Auffassung der DRB als auch nach Auffassung der NRV der entscheidende Grund dafür, dass nur eine wirklich unabhängige Organisation der Justiz Unabhängigkeit und Gesetzesbindung der Richter gewährleisten kann. Die Organisation der Justiz muss von der Exekutive vollständig abgekoppelt werden; die Justiz muss sich selbst verwalten. In den meisten Ländern Europas ist dieser Grundgedanke - anders als in Deutschland - sowohl in der staatsrechtlichen Wissenschaft als auch im allgemeinen politischen Bewusstsein weitgehend anerkannt.

d) Wo Menschen arbeiten, gibt es immer Fehler, Unzulänglichkeiten und Machtmissbrauch. Das gilt für eine - zukünftige - selbstverwaltete Justiz in Deutschland grundsätzlich im Ansatz genauso wie für die derzeit von der Exekutive organisatorisch gesteuerte und (fremd) verwaltete Justiz. Entscheidend ist, dass eine selbst verwaltete Justiz die Chancen für weniger Fehler und weniger Machtmissbrauch verbessern wird:

- Eine selbst verwaltete Justiz verringert die Abhängigkeiten der Richter (z. B. bei Fragen der „Karriere“ und bei Fragen der Ressourcenzuteilung für die einzelnen Gerichte). Weniger Abhängigkeit bedeutet weniger (gesetzesfreies) Anpassungsverhalten der Richter und Verbesserung korrekter Gesetzesanwendung.

- Derzeit wird die Justiz in Deutschland von der Exekutive (Landesregierungen bzw. Justizminister) organisiert. Die Exekutive verfolgt immer bestimmte politische Ziele. Die Organisation der Justiz durch die Exekutive bedeutet immer eine Steuerung der Justiz in Richtung der politischen Ziele der Exekutive. Nur eine Selbstverwaltung kann die Justiz von einer Steuerung in Richtung bestimmter Zielsetzungen der Exekutive befreien. Die Selbstverwaltung der Justiz ist notwendige Machtbegrenzung der Exekutive.

e) „Sine spe ac metu“ ist für die NRV die Überschrift, die für alle Konzepte zur Selbstverwaltung maßgeblich ist. Nur dann, wenn wir Richter uns bewusst werden, wie Hoffnungen und Befürchtungen (,spes und metus“) unser Verhalten und unser Denken auch bei richterlichen Entscheidungen maßgeblich beeinflussen können, gewinnt der Gedanke der Selbstverwaltung einen entscheidenden Sinn. Diese - letztlich auch richterkritische - Sichtweise wird im DRB wohl nicht vollständig geteilt.

\section{Selbstverwaltung von unten im Konzept der NRV}

Das Konzept der NRV unterscheidet sich von dem des DRB insofern wesentlich, als nicht nur eine neue bürokratische Zuständigkeit über den „Justizverwaltungsrat“ bzw. über den ,Justizrat““ anstelle des Justizministeriums geschaffen werden soll, sondern die gestaltende Eigenverantwortung der Richterinnen und Richter im Sinne des demokratischen grundgesetzlichen Auftrags über organisatorische Zuständigkeiten gefördert werden soll. Erforderlich ist ein System, das dem Selbstverwaltungsanspruch des Grundgesetzes zugunsten der kleinstmöglichen Einheit (vgl. insbesondere Art. 28 Abs. 2 GG) folgend, die Eigenverantwortung aller Richterinnen und Richter unmittelbar 
an ihrem Arbeitsplatz aufruft, so dass sie in gemeinsamer Verantwortung alle Abläufe am jeweiligen Gericht selbst gestalten können. Nur so werden nach Überzeugung der NRV die besten Voraussetzungen für eine möglichst effiziente, qualitativ anspruchsvolle und unabhängige Rechtsprechung im Sinne des grundgesetzlichen Auftrags geschaffen.

Aus diesem Ansatz ergibt sich: Für die Verwaltung der einzelnen Gerichte sollen nach den Vorstellungen der NRV die Präsidien der Gerichte verantwortlich sein. Der von den Richtern des jeweiligen Gerichts gewählte Präsident ist dem Präsidium gegenüber verantwortlich und im Verhältnis zum Präsidium dessen ausführendes Organ. Demgegenüber möchte der DRB an einem hierarchischen Aufbau der Justizverwaltung festhalten. Der Justizverwaltungsrat ist im Modell des DRB (dem heutigen Justizministerium vergleichbar) die oberste Justizbehörde, der die nachgeordneten Präsidenten der Gerichte (wie heute im Verhältnis zum Ministerium) unterstehen.

\section{Bundesgesetz oder Landesgesetz?}

Der DRB schlägt zur Selbstverwaltung eine Lösung vor, die durch Gesetze auf Landesebene umgesetzt werden soll, während die NRV ein Bundesgesetz für vorzugswürdig hält. Der DRB-Entwurf kommt im Übrigen ohne Grundgesetzänderung aus, während nach Auffassung der NRV zur Durchführung der Selbstverwaltung bestimmte Änderungen des Grundgesetzes erfolgen sollten.

4. Teilweise unterschiedliche Funktionen der Selbstverwaltungsgremien in den Entwürfen von NRV und DRB

Das DRB-Modell sieht als Selbstverwaltungsgremien einen „Justizwahlausschuss“ und einen „Justizverwaltungsrat““ vor. Im NRV-Modell gibt es einen „Richterwahlausschuss“ und einen „Justizrat“. Die Gremien entsprechen sich teilweise. Zum Teil gibt es allerdings auch wesentliche Unterschiede in den Funktionen der Gremien.

a) Beiden Modellen gemeinsam ist die Vorstellung, dass die Organisations-Aufgaben des heutigen Justizministeriums in Zukunft weitgehend auf einen ,Justizverwaltungsrat“ (DRB) bzw. „Justizrat“ (NRV) übergehen. Hier liegt ein Kern der Selbstverwaltungsidee. „Justizverwaltungsrat" bzw. „Justizrat" sind Organe, die - anders als die heutigen Justizministerien - organisatorisch nicht zur Exekutive gehören.

b) Während beim DRB der „Justizwahlausschuss“ ein reines Wahlgremium ist (Wahl der Mitglieder des Justizverwaltungsrats), hat der „Richterwahlausschuss“ im NRVModell eigene sachliche Aufgaben. Der „Richterwahlausschuss“ der NRV ist für die Einstellung von Richtern verantwortlich. Das NRV-Modell steht beim Richterwahlausschuss der heutigen Regelung im Grundgesetz (Art. 98 Abs. 4 GG) näher als das DRBModell.

c) Nach Auffassung der NRV wird durch den „Richterwahlausschuss“ im NRV-Modell eine demokratische Legitimation der Justiz besser verwirklicht als im Modell des DRB. Der „Richterwahlausschuss“ besteht zu 2/3 aus Mitgliedern des jeweiligen Landesparlaments. Damit hat die Legislative im NRV-Modell einen entscheidenden Einfluss auf die Auswahl und die Einstellung von Richtern. Der Einfluss der Legislative 
auf die Auswahl und Einstellung von Richtern durch den „Justizverwaltungsrat“ im DRB-Modell ist schwächer und indirekter.

d) Im ,Justizrat“ der NRV (der allerdings für die Einstellung von Richtern nicht zuständig ist, s. o.), ist der Gedanke der Selbstverwaltung der Justiz konsequenter verwirklicht als im „Justizverwaltungsrat“ des DRB. Die Richter haben im NRV-Modell einen größeren Einfluss auf die Zusammensetzung des „Justizrats“ als im DRB-Modell auf die Zusammensetzung des „Justizverwaltungsrats“.

\section{Hierarchiefreiheit - Keine Beförderungen mehr im NRV-Modell}

Im NRV-Modell spielt - anders als im Konzept des DRB - der Abbau von Binnenhierarchien in der Justiz eine wesentliche Rolle. Die NRV ist der Überzeugung, dass Hierarchien Abhängigkeiten schaffen, die es zu beseitigen gilt. Im NRV-Modell gibt es keine Beförderungen mehr, sondern nur noch Versetzungen. Alle Richter (Richter am Amtsgericht, Richter am Landgericht, Richter am Oberlandesgericht und Vorsitzende Richter an den verschiedenen Gerichten) stehen auf der selben Hierarchiestufe und werden gleich bezahlt. Unterschiedliche Gehaltsstufen ergeben sich nur noch aus einem unterschiedlichen Lebensalter der Richter. Die NRV sieht das Streben nach Beförderungen und die damit verbundenen psychologischen Mechanismen als ein Hindernis an für eine selbstbewusste, nur am Gesetz orientierte Tätigkeit der Richter. Der DRB will demgegenüber am bisherigen System von Beurteilungen und Beförderungen in der Justiz grundsätzlich festhalten. Die Idee der Hierarchiefreiheit (gleiche Bezahlung für verschiedene Richterämter) ist in einigen anderen europäischen Ländern bereits realisiert. 